\title{
Spin manipulation in Co-doped ZnO
}

\author{
Heidemarie Schmidt ${ }^{1, a}$ \\ ${ }^{1}$ Forschungszentrum Dresden-Rossendorf e.V., Bautzner Landstrasse 400, 01328 Dresden, \\ Germany \\ ${ }^{a}$ Heidemarie.Schmidt@fzd.de
}

\begin{abstract}
Keywords: magnetic $\mathrm{ZnO}$, n-type conducting, s-d exchange coupling, spin-splitted conduction band, magnetotransport
\end{abstract}

\begin{abstract}
The magnetoresistance of n-type conducting, paramagnetic Co-doped $\mathrm{ZnO}$ films prepared by pulsed laser deposition on sapphire substrates has been studied experimentally and theoretically. Positive magnetoresistance (MR) of $124 \%$ has been observed in the film with the lowest electron concentration of $8.3 \cdot 10^{17} \mathrm{~cm}^{-3}$, while only a negative MR of $-1.9 \%$ was observed in the film with an electron concentration of $9.9 \cdot 10^{19} \mathrm{~cm}^{-3}$ at $5 \mathrm{~K}$. The positive MR is attributed to the quantum correction on the conductivity due to the $s$ - $d$ exchange interaction induced spin splitting of the conduction band. The negative MR is attributed to the magnetic field suppressed weak localization [1]. Voltage control of the electron concentration in Schottky diodes revealed a drastic change of the magnetoresistance and demonstrated the electrically controllable magnetotransport behavior in Codoped $\mathrm{ZnO}$ [2]. The magnetically controllable spin polarization in Co-doped $\mathrm{ZnO}$ has been demonstrated at $5 \mathrm{~K}$ in magnetic tunnel junctions with Co-doped $\mathrm{ZnO}$ as a bottom electrode and Co as a top electrode [3]. There spin-polarized electrons were injected from Co-doped $\mathrm{ZnO}$ to a crystallized $\mathrm{Al}_{2} \mathrm{O}_{3}$ layer and tunnelled through an amorphous $\mathrm{Al}_{2} \mathrm{O}_{3}$ barrier. Our studies demonstrate the spin polarization and manipulation in Co-doped $\mathrm{ZnO}$.
\end{abstract}

Introduction. Spintronics is related to the manipulation of spins. It mainly consists of three processes, spin generation, spin transportation, and spin detection. Diluted magnetic $\mathrm{ZnO}$ can be realized by epitaxial growth on transparent substrates using pulsed laser deposition (PLD). A ferromagnetic coupling of the magnetic moments localized at cation lattice sites has been theoretically predicted for p-type conducting $\mathrm{ZnO}$ doped with magnetic ions [4]. However, typically $1 \mu \mathrm{m}$ thick magnetic $\mathrm{ZnO}$ films are intrinsically n-type conducting or insulating and contain up to 10 at.\% magnetic ions being isovalently incorporated on cation lattice sites. The magnetic properties of $\mathrm{ZnO}$ depend on the hybridization between the magnetic ions or intrinsic defects and the charge carriers at the Fermi edge, thus they depend on the thin film growth conditions. Weak ferromagnetism has been probed in insulating $\mathrm{Mn}$-doped $\mathrm{ZnO}$ thin films, in n-type conducting, partially compensated $\mathrm{Mn}$-doped $\mathrm{ZnO}$ thin films and in n-type conducting, partially compensated $\mathrm{ZnO}$ thin films without magnetic ions. Space charge spectroscopy has been used to reveal acceptorlike defects in the n-type conducting, partially compensated, ferromagnetic thin films, i.e. acceptorlike defects mediate the weak ferromagnetism probed in Mn-doped $\mathrm{ZnO}$. The capture cross section of deep traps for charge carriers is smaller in magnetic films compared to unmagnetic ones. Energetically deep lying defects in $\mathrm{ZnO}$ follow the Meyer-Neldel rule, which describes the entropy change during charging of deep electron defects, with the isokinetic temperature amounting to 226 $\mathrm{K}$ [5]. X-ray magnetic circular dichroism measurements at $30 \mathrm{~K}$ confirmed the paramagnetic properties of Co-doped $\mathrm{ZnO}$ films [6]. Magnetoresistance changes in n-type conducting, magnetic $\mathrm{ZnO}$ films with different magnetic species and concentrations of free charge carriers have been systematically investigated as a function of an external magnetic field with regard to future applications in magnetoresistive $\mathrm{ZnO}$-based spintronics devices. Especially, at the metal-insulator transition, which has been determined to lie at $\mathrm{n}_{\mathrm{c}}=4.9 \times 10^{19} \mathrm{~cm}^{-3}$ in $\mathrm{ZnO}$ thin films [7], the magnetoresistance can be sensitively controlled. Modelling the positive magnetoresistance in $\mathrm{ZnO}$ :Co thin films being superimposed by a weak negative magnetoresistance was possible for the 
strong $\left(\mathrm{n}<\mathrm{n}_{\mathrm{c}}\right)$ and weak $\left(\mathrm{n}>\mathrm{n}_{\mathrm{c}}\right)$ localization regime [1]. In this paper we will focus on the spinsplitting of the conduction band due to sd-exchange interaction between free charge carriers and localized magnetic moments, the electrically controllable magnetoresistance and magnetically controllable spin polarization in Co-doped $\mathrm{ZnO}$ thin films, Schottky diodes and magnetic tunnel junctions, respectively.

Experimental Results and Discussion. Magnetotransport measurements in the classical van-derPauw geometry represent a fast and precise method for investigating conducting, magnetic thin films. In $\mathrm{n}$ - or $\mathrm{p}$-conducting $\mathrm{ZnO}$ the $\mathrm{s}-(3 \mathrm{~d}, 4 \mathrm{f})$ or $\mathrm{p}-(3 \mathrm{~d}, 4 \mathrm{f})$ exchange interaction, respectively, leads to spin polarization and may cause positive magnetoresistance. We modeled the corresponding spin polarization from the magnetotransport data. However, because of different mechanisms influencing the magnetotransport properties, superimposed positive and negative magnetoresistance may be observed in dependence on temperature and magnetic field strength. An advanced magnetotransport modelling approach should combine different mechanisms for example magnetic polarons and electron localization causing negative magnetoresistance and s-d and s-f induced spin splitting of the conduction band causing positive magnetoresistance in magnetic, $\mathrm{n}$-type conducting $\mathrm{ZnO}$ films.

Spin-splitting of the Conduction Band. We modeled the spin polarization in $\mathrm{ZnO}: \mathrm{Co}$ and ZnO:Mn from the decrease of positive magnetoresistance with the free electron concentration [8]. Positive magnetoresistance has been interpreted in terms of the influence of the sd-exchange interaction on the disorder-modified electron-electron interactions $[9,10]$ and may be attributed to magnetic scattering of spin polarized free electrons. The electron concentration of spin up $\uparrow$ or spin down $\downarrow$ electrons can be calculated by:

$$
\int_{E c}^{\infty} \frac{2 \pi}{\hbar^{3}}\left(2 m^{*}\right)^{\frac{3}{2}} E^{\frac{1}{2}}\left(1+\exp \left(\left(E-E_{f}\right) / k_{B} T\right)\right)^{-1} d E,
$$

where $\mathrm{m}^{*}$ is the effective mass of electrons, $\mathrm{E}_{\mathrm{f}}$ the Fermi energy, $\mathrm{E}_{\mathrm{c}}$ is the bottom of the spin up $\uparrow$ or spin down $\downarrow$ conduction band, respectively, and $\mathrm{k}_{\mathrm{B}}$ the Boltzmann constant. Due to the splitting of the conduction band, there is an energy gap $\delta$ between the spin-up and spin-down polarized conduction band (Fig. 1a). The position of the Fermi level $\mathrm{E}_{\mathrm{f}}$ was adjusted to obtain the electron concentration $n$, which equals $n \uparrow+n \downarrow$.

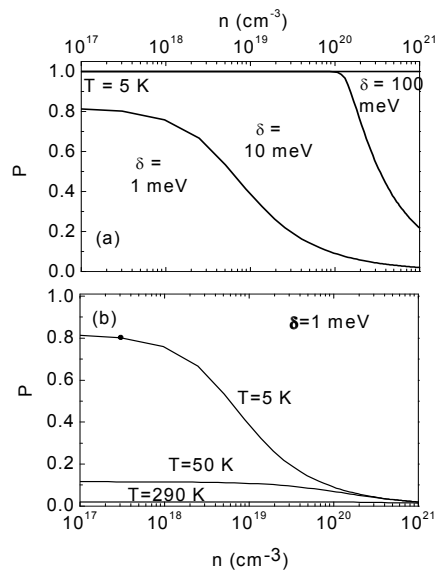

Fig. 1 (a) Simulated spin polarization $\mathrm{P}$ in dependence on the electron concentration $\mathrm{n}$ at $5 \mathrm{~K}$ for different conduction band splittings $\delta$ and (b) at different temperatures for $\delta=1 \mathrm{meV}$. We use $\mathrm{m}^{*}=0.24 \mathrm{~m}_{0}$ as the effective electron mass in $\mathrm{ZnO}$. The modelling has been performed under the assumption that the free electron model is valid for $\mathrm{n}$ ranging from $10^{17}$ to $10^{20} \mathrm{~cm}^{-3}[8]$. 
There exists no experimentally determined value for the conduction band splitting $\delta$ in magnetic $\mathrm{ZnO}$ and we use 1, 10, and $100 \mathrm{meV}$ as initial values for modelling the spin polarization $\mathrm{P}$ (Fig. 1(a)). P is defined by $(n \uparrow-n \downarrow) /(n \uparrow+n \downarrow)$. For $\delta=100 \mathrm{meV}, \mathrm{P}$ is nearly independent on $\mathrm{n}$. For $\delta=10$ $\mathrm{meV}$, P keeps 1 for $\mathrm{n}<10^{20} \mathrm{~cm}^{-3}$ and drops with $\mathrm{n}$ increasing above $10^{20} \mathrm{~cm}^{-3}$. For $\delta=1 \mathrm{meV}, \mathrm{P}$ continuously decreases with increasing $\mathrm{n}$ and almost drops to zero for $\mathrm{n}>10^{20} \mathrm{~cm}^{-3}$. By comparing the dependence of the simulated spin polarization $\mathrm{P}$ and of the measured positive magnetoresistance on temperature and electron concentration, we determined a splitting of the conduction band $\mathrm{E}_{\mathrm{c}}$ in $\mathrm{ZnO}: \mathrm{Co}$ and $\mathrm{ZnO}: \mathrm{Mn}$ below $10 \mathrm{meV}$ [8].

Electrically Controllable Magnetoresistance. Diluted magnetic semiconductors provide the capability of controlling the charge and spin of the charge carriers simultaneously. The clear understanding of the magnetotransport properties of DMS in an external magnetic and electric field is important for future spintronics applications. A $\mathrm{Zn}_{0.96} \mathrm{Co}_{0.04} \mathrm{O}$ film with low electron concentration (about $1.5 \times 10^{17} \mathrm{~cm}^{-3}$ at $21 \mathrm{~K}$ ) on a highly conducting $\mathrm{Zn}_{0.99} \mathrm{Al}_{0.01} \mathrm{O}$ layer has been deposited on an $a$-plane sapphire substrate by PLD [2]. To study the magnetoresistance of depleted, highly insulating Co-doped $\mathrm{ZnO}$ an $\mathrm{Au}$ ohmic contact and a Pd Schottky contact were deposited on the $\mathrm{Zn}_{0.99} \mathrm{Al}_{0.01} \mathrm{O}$ and $\mathrm{Zn}_{0.96} \mathrm{Co}_{0.04} \mathrm{O}$ layer, respectively. Positive magnetoresistance of $30 \%$ with a current of $10^{-6} \mathrm{~A}$ was observed at $5 \mathrm{~K}$ (Fig. 2). The positive MR decreases drastically at $5 \mathrm{~K}$ and changes to negative MR at $50 \mathrm{~K}$ with increasing current, which is considered to be due to the bias voltage control of the electron concentration in the $\mathrm{Zn}_{0.96} \mathrm{Co}_{0.04} \mathrm{O}$ layer. The concentration of free electrons in the undepleted Co-doped $\mathrm{ZnO}$ has been measured by capacitance-voltage measurements and amounts to $1.5 \times 10^{17} \mathrm{~cm}^{-3}$ at $21 \mathrm{~K}$. The concentration of remaining free electrons in the depleted Co-doped $\mathrm{ZnO}$ has been estimated to be about $3 \times 10^{13} \mathrm{~cm}^{-3}$ from the correspondingly measured resistivity (about $90 \Omega \cdot \mathrm{m}$ measured at $5 \mathrm{~K}$ with $1 \mu \mathrm{A}$ ) and using the electron mobility in Co-doped $\mathrm{ZnO}[7]$.

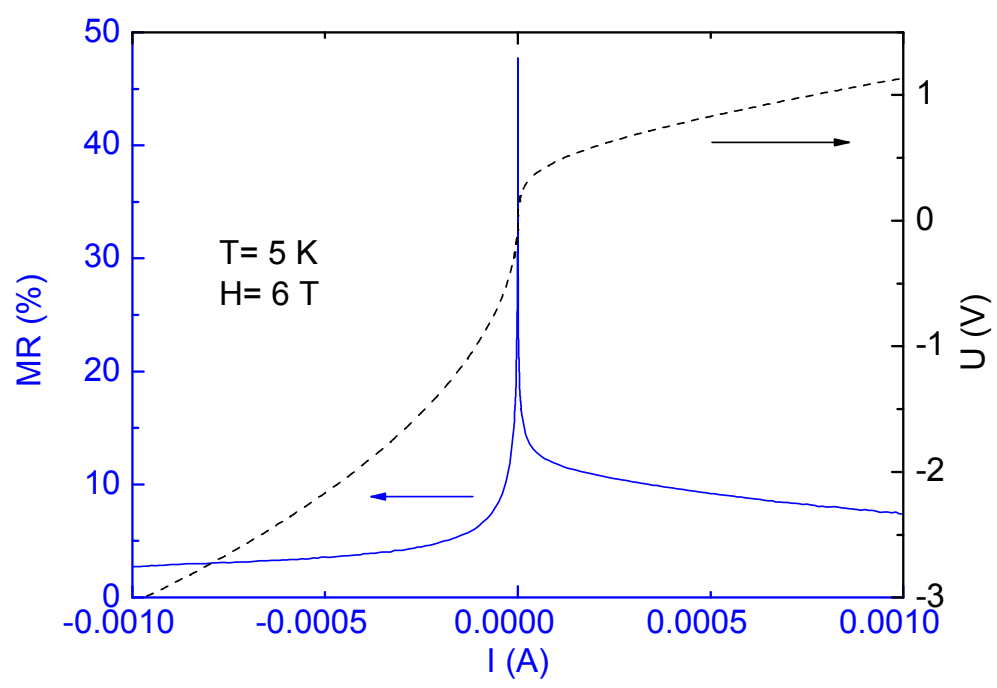

Fig. 2 The current dependent MR (solid line) and the IV characteristics (dashed line) measured at 5 $\mathrm{K}$ and $6 \mathrm{~T}$.

This work demonstrates the electrically controllable magnetotransport behavior in Co-doped $\mathrm{ZnO}$ Schottky diodes due to the electrically controllable electron concentration $\mathrm{n}$ and the dependence of the magnetoresistance in Co-doped $\mathrm{ZnO}$ on $\mathrm{n}$ [2].

Magnetically Controllable Magnetoresistance. Future developments will also tackle the exploitation of magnetic oxides in magnetic random access memory (MRAM) devices based on 
tunnel magnetoresistance (TMR) structures where the magnetization can be switched by an external magnetic field. The magnetically controllable spin polarization in Co-doped $\mathrm{ZnO}$ has been demonstrated at $5 \mathrm{~K}$ in magnetic tunnel junctions with Co-doped $\mathrm{ZnO}$ as a bottom electrode and Co as a top electrode on a nonconducting substrate [3]. Before depositing the Co-doped $\mathrm{ZnO}$ bottom electrode, we deposited an Al-doped $\mathrm{ZnO}$ layer as a buffer layer on the sapphire substrate. At low field the small negative magnetoresistance (MR) of the bottom electrode (not shown here) might originate from the Al-doped $\mathrm{ZnO}$ [9]. However, the deposition parameters of Co-doped $\mathrm{ZnO}$ were chosen to prepare the highly conductive film, which will also lead to the low field negative MR [7]. The positive MR at intermediate field and negative MR at high fields originate from the Co-doped $\mathrm{ZnO}$ [7]. In our junction structure, the current will then go perpendicular to the film through the $\mathrm{Al}_{2} \mathrm{O}_{3}$ barrier layer to the Co top electrode. The mainly interesting phenomenon is the low field butterfly positive MR behaviour, as shown in Fig. 3. Contradicted to the normally observed TMR effect, double peaks have been observed in each swept curve which are located at both sides of $0 \mathrm{~T}$. As one can see, with increasing applying current, the MR effect becomes weaker, which is typical TMR effect [11]. With applying $1 \mu \mathrm{A}$, the butterfly positive MR disappears, and the junction was broken, and no butterfly positive MR at low field can be observed as shown in Fig. 2(d). After the application of $1 \mu \mathrm{A}$, no butterfly positive MR at low field can be observed with smaller current. As an example the first $\mathrm{ZnO}$-based TMR structure revealing magnetic field switching is shown in Fig. 3.

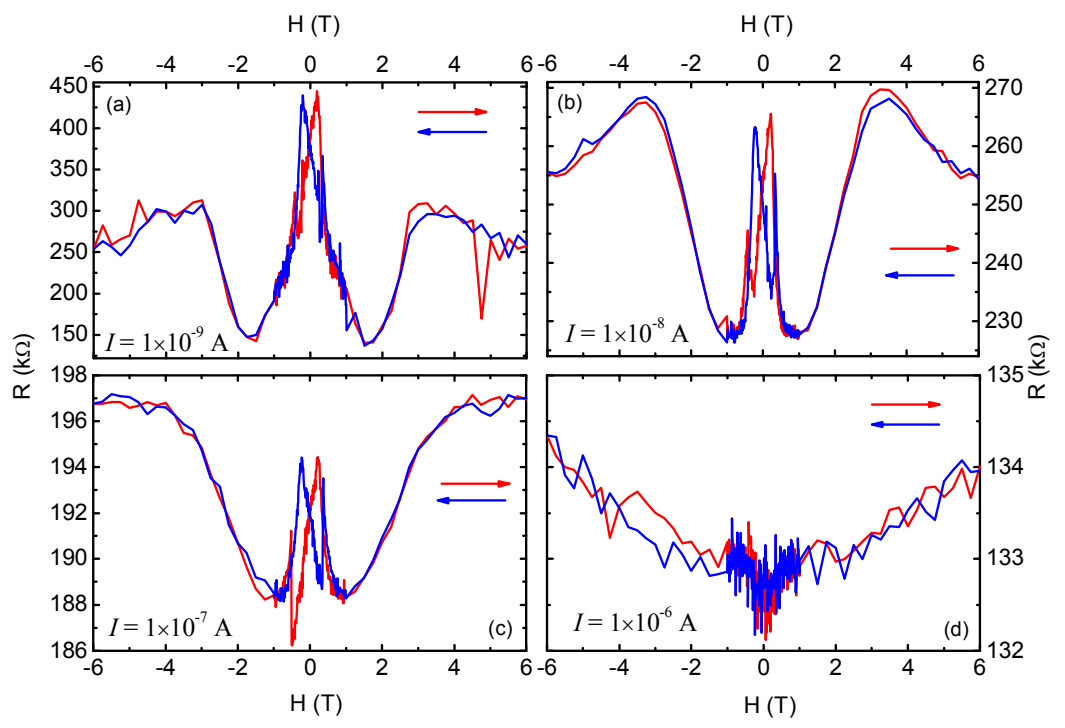

Fig. 3 Field dependent resistance of the TMR sample ( $\mathrm{ZnCoO}$ bottom electrode, crystalline/amorphous $\mathrm{Al}_{2} \mathrm{O}_{3}$ barrier, Co top electrode) measured at $5 \mathrm{~K}$ with field applied in the film plane with the higher voltage applied on the Co top electrode and lower voltage on the $\mathrm{ZnO}$ bottom electrode for different constant currents. The applied constant current is (a) $1 \times 10^{-9} \mathrm{~A}$, (b) $1 \times 10^{-8} \mathrm{~A}$, (c) $1 \times 10^{-7} \mathrm{~A},(\mathrm{~d}) 1 \times 10^{-6} \mathrm{~A}$. The arrows show the field sweep direction, from $-6 \mathrm{~T}$ to $6 \mathrm{~T}$ (red) and from $6 \mathrm{~T}$ to $-6 \mathrm{~T}$ (blue) [3].

The MR curves at high field $(\mathrm{H}>1 \mathrm{~T})$ are very similar to the MR curves probed on $\mathrm{ZnCoO}$ thin films. The low field butterfly positive MR behavior reveals double peaks located at both sides of 0 $\mathrm{T}$ with the lower peak at the starting field and the higher peak at the end field for each sweep direction. When applying a current of $1 \mu \mathrm{A}$ the junction was destroyed and the butterfly positive MR disappears [3]. Note that novel MRAM devices based on TMR structures reveal currentinduced switching of magnetization. 
Summary and outlook. Insulating and n-type conducting, diluted magnetic $\mathrm{ZnO}$ films have been prepared by pulsed laser deposition and the electric and magnetic controllability of magnetoresistance has been demonstrated. The magnetotransport properties of magnetic $\mathrm{ZnO}$ depend on the species and concentration of magnetic ions, the concentration of free charge carriers and the film thickness. The large positive magnetoresistance in n-type conducting $\mathrm{ZnCoO}$ is attributed to the quantum correction on the conductivity due to the sd-exchange interaction induced spin-splitting of the conduction band. The sd-exchange induced splitting of the conduction band being proportional to the exchange coupling constant $\alpha$ is less than $10 \mathrm{meV}$. By comparing the exchange coupling constants $\alpha$ and $\beta$ in II-VI compound semiconductors it is expected that the pdexchange interaction induced splitting of the valence band being proportional to the exchange coupling constant $\beta$ is larger than $30 \mathrm{meV}$, thus producing a finite spin polarization in $\mathrm{p}$-type conducting, magnetic $\mathrm{ZnO}$ even above room temperature. Room temperature ferromagnetism in diluted magnetic $\mathrm{ZnO}$ could pave the way to exploit the spin in addition to charge in future $\mathrm{ZnO}$ based spinelectronics devices. Because Co ions are isovalent dopants in $\mathrm{ZnO}$, additional acceptor dopants have to be added, e.g. by implanation, and electrically activated by pulsed laser annealing under oxygen overpressure, otherwise oxygen tends to evaporate from the $\mathrm{ZnO}$ surface. Therefore, the incorporation of acceptor dopants above their solubility limit is the main challenge for the fabrication of ferromagnetic $\mathrm{ZnO}$. Because $\mathrm{Co}$ and $\mathrm{Mn}$ shows a very low diffusity in $\mathrm{ZnO}$ over the entire temperature range, from a themodynamical point of view p-type conducting Co- and $\mathrm{Mn}$ doped $\mathrm{ZnO}$ spintronics devices will be stable enough for room temperature applications [12]. The thermodynamic stability of laser annealed p-type conducting magnetic semiconductors has already been proven by the hysteretic magnetotransport of spin-polarized holes in accordance with magnetization below the Curie temperature in Mn-doped Ge [13] and in Mn-doped GaAs [14].

\section{References}

[1] Q. Xu, L. Hartmann, H. Schmidt, H. Hochmuth, M. Lorenz, D. Spemann and M. Grundmann, Phys. Rev. B 76 (2007), p. 134417

[2] Q. Xu, S. Zhou, D. Bürger, H. Hochmuth, M. Lorenz, M. Grundmann and H. Schmidt, Jpn. J. of Appl. Phys. 49 (2010), p. 043002

[3] Q. Xu, L. Hartmann, S. Zhou, A. Mücklich, M. Helm, G. Biehne, H. Hochmuth, M. Lorenz, M. Grundmann and H. Schmidt, Phys. Rev. Lett. 101 (2008), p. 076601

[4] T. Dietl, H. Ohno, F. Matsukura, J. Cibert, D. Ferrand, Science 287 (2000), p. 1019

[5] H. Schmidt, M. Wiebe, B. Dittes, M. Grundmann, Appl. Phys. Lett. 91 (2007), p. 232110

[6] Q. Xu, S. Zhou, D. Marko, K. Potzger J. Fassbender, M. Vinnichenko, M. Helm, H. Hochmuth, M. Lorenz, M. Grundmann, H. Schmidt, J. of Phys. D: Appl. Phys. 42 (2009), p. 085001

[7] Q. Xu, L. Hartmann, H. Schmidt, H. Hochmuth, M. Lorenz, R. Schmidt-Grund, C. Sturm, D. Spemann, M. Grundmann, Phys. Rev. B 73 (2006), p. 205342

[8] Q. Xu, L. Hartmann, H. Schmidt, H. Hochmuth, M. Lorenz, R. Schmidt-Grund, C. Sturm, D. Spemann, M. Grundmann, Y. Liu, J. Appl. Phys. 101 (2007), p. 063918

[9] T. Fukumura, Zhengwu Jin, A. Ohtomo, H. Koinuma, M. Kawasaki, Appl. Phys. Lett. 75 (1999), p. 3366 
[10] T. Andrearczyk, J. Jaroszyński, G. Grabecki, T. Dietl, T. Fukumura, M. Kawasaki, Phys. Rev. B 72 (2005), p. 121309(R)

[11] J. S. Moodera, Lisa R. Kinder, Terrilyn M. Wong, R. Meservey, Phys. Rev. Lett. 74 (1995) p. 3273

[12] D. Bürger, M. Seeger, S. Zhou, I. Skorupa, M. Helm, H. Schmidt, submitted.

[13] S. Zhou, D. Bürger, C. Baumgart, W. Skorupa, C. Timm, P. Oesterlin, M. Helm, H. Schmidt, Phys. Rev. B 81 (2010), p. 165204

[14] D. Bürger, S. Zhou, M. Pandey, C.S. Viswanadham, J. Grenzer, O. Roshchupkina, W.

Anwand, H. Reuther, V. Gottschalch, M. Helm, H. Schmidt, Phys. Rev. B 81 (2010), p. 115202 